\title{
ANALYSIS OF TWEETS RELATED WITH PHYSICAL ACTIVITY DURING COVID-19 OUTBREAK
}

\section{Tolga Şahin ${ }^{1}$, Hikmet Gümüş ${ }^{1}$, Celal Gençoğlu ${ }^{1}$}

\author{
${ }^{1}$ Faculty of Sport Sciences, Dokuz Eylül University, Izmir, Turkey. \\ Address for Correspondence: Celal Gençoğlu, E-mail: celal.gencoglu@deu.edu.tr \\ Received: 28.01.2021; Accepted: 17.02.2021; Available Online Date: 04.03.2021 \\ @C Copyright 2021 by Dokuz Eylül University, Institute of Health Sciences - Available online at https://dergipark.org.tr/en/pub/jbachs
}

Cite this article as: Sahin T, Gumus H, Gencoglu C. Analysis of Tweets Related with Physical Activity During COVID-19 Outbreak. J Basic Clin Health Sci 2021; 1: 42- 48.

\begin{abstract}
Purpose: The COVID-19 pandemic affected the daily lifestyle and habits around the worldwide. Besides the impact of coronavirus on social life and canceling of gyms limited physical activity and exercise. The aim of this study was to analysis of tweet numbers and attitudes related with physical activity during the COVID-19 pandemic regarding the timeline of Turkey outbreak.

Methods: Twitter query performed in Turkish tweets between 1st of January and 14th of May 2020 including keywords "exercise" or "physical or activity" or "fitness" or "plates" or "gymnastics" or "inactive". Collected tweet data sent to Google cloud natural language API for estimation of sentiment score. Tweets assigned as non-COVID period (1st of Jan-14th Mar; $n=74$ days) and COVID period (14th Mar-14th May; $\mathrm{n}=61$ days) according to the prognosis of the pandemic in Turkey.

Results: The number of tweets and both positive and negative sentiments score of tweets significantly increased in the COVID period in comparison to the non-COVID period. There was a significant decreased sentiment scores in COVID period when compared to non-COVID period however, during the COVID period, no significant differences found between forced isolation days and non-forced isolation days.

Discussion: These results suggest that people had more leisure time and realized the importance of being active for a healthy lifestyle during this lockdown period in Turkey then both performed exercises and posted to Twitter more often. Current findings also showed that social media is a platform for promoting physical activity and exercise via peoples' necessity to share and get interaction.
\end{abstract}

Keywords: COVID-19 pandemic, lockdown, physical activity, sentiment analysis.

\section{INTRODUCTION}

The new coronavirus-related epidemic began in Wuhan city, China in December 2019 and spread the other countries in different timelines. The spread out of COVID-19 start with positive diagnoses of a person who traveled abroad from Turkey on 10th March than first Turkish citizens identified as infected on 13th March. As shown in Figure 1a, the timeline consists of that Turkish government's first time put in the quarantine of a group of people In 15th Mar, the first nationwide lockdown on 11th Apr, and the peak number of new cases on 12th Apr (1). The COVID-19 
pandemic affected the customary lifestyle of almost every area such as education (2), traveling, and tourism, economy (3), and forced people to changing daily habits. Besides the impact of coronavirus on social life and canceling of gyms limited physical activity and exercise. Social distancing stay at home advice and strict quarantine periods lead to diminishing energy expenditure (4). Recently, researchers showed an increased interest in behavioral changes such as sleep, nutrition, work, physical activity, and exercise during unexpectedly lockdown period. In a study investigating the relationship between digital media usage and sleep pattern in the lockdown, Cellini et al. showed that increased using digital media near bedtime, however no significant change in the sleep routine (5). A recent study on the limitation of physical activity during COVID-19 pandemic observed that a reduced number of steps in children with Congenital Heart Disease (6). Previous studies indicated that social media interventions are a promising technology to monitoring health status and daily activity and also encourage physical activity $(7,8)$. Nevertheless, COVID-19 preventions such as nationwide lockdown and strict quarantine may result in potential side effects such as weight gain, metabolic disorders, hypertension i.e. $(9,10)$. In this context, it suggests the beneficial effects of social media usage to prevent sedentary behavior and inactivity during COVID-19 outbreak and lockdown (11). One of the key methods of investigation to efficacy and mechanism of social media influence is sentiment analysis. The sentiment analysis uses the interpretation and classification of positive, negative, and neutral attitude within the posted text data (i.e. hashtag \#) using text analysis techniques (12). One study investigated the sentiment analysis of the sharing from Twitter after the declaration of lockdown in India. They found that lockdown decision gets a positive reflection from the Indians however also exposed sadness and worry sentiment in the social media (13). Harris et al. conducted a descriptive study on Twitter messages hashtagged "\#thinspo and \#fitspo" and found most of the tweets related to dieting and focused on thin body image (14). In view of social media usage and physical activity and health relation, the social platform data seems to provide a meaningful reference cause people to post to social media what they do or eat. Sentiment analysis induces to enable generalized and measurable data from social media platforms. As our best knowledge, there is no social media sentiment analysis study considered following the COVID-19 outbreak. Therefore, the aim of this study was to analysis of tweet numbers and attitudes related with physical activity during the COVID-19 pandemic regarding the timeline of Turkey outbreak.

\section{METHODS}

Twitter query performed in Turkish tweets between 1st of January and 14th of May 2020 including keywords "exercise" or "physical or activity" or "fitness" or "plates" or "gymnastics" or "inactive". The custom written script with Python language used to get data and process. Natural Language Toolkit library built for the Preprocessing stage to achieve precise sentiment analysis. attained tweet data was scraped. In this process, retweets removed, usernames took out, and stopwords (i.e. one, anymore, one more) excluded by using a list of Turkish stopwords. After tokenized tweets, they sent to the Google cloud natural language API for estimation of sentiment score. Sentiment score differs between -1 and +1 which -1 and -0.25 considered negative sentiment score, -0.25 and +0.25 is neutral and +0.25 and +1 is a positive one. The processed tweets and sentiment scores sent and stored in MySql . The data receiving and preparation algorithm showed in Figure 1.

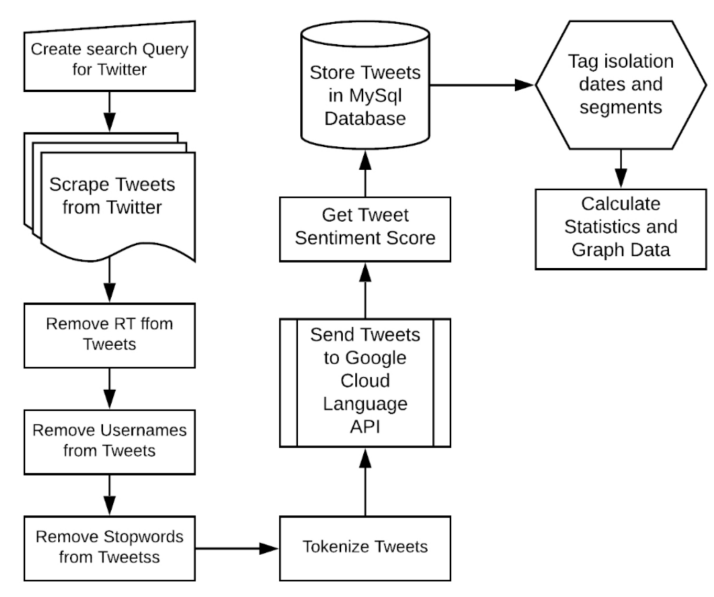

Figure 1. The flow structure for tweet classification and sentiment analysis

\section{Statistical analysis}

Statistical analysis

Tweets assigned as non-COVID period (1st of Jan14th Mar; $n=74$ days) and COVID period (14th Mar14th May; $n=61$ days) according to the prognosis of the pandemic in Turkey. Also, another designation 
performed to the tweets in COVID period by forced isolation and non-forced isolation days in Turkey.

Statistical analysis was performed using SPSS 25 software (IBM Corp. Released in 2017. IBM SPSS Statistics for Windows, Version 25.0. Armonk, NY). A nonparametric Mann Whitney $U$ test was used to analyze number of totals, negative and positive tweets, mean sentiment scores of negative tweets and mean sentiment scores of total tweets between forced isolation days $(n=11)$ and non-forced isolation days $(n=50)$ within the COVID period. Neutral tweets were excluded from the analysis of sentiment scores of positive and negative tweets. Comparison of the number of positive tweets, negative tweets, total tweets, mean sentiment scores of positive tweets, mean sentiment scores of negative tweets and mean sentiment scores of total tweets between the COVID period and non-COVID period were analyzed with independent t-test. Linear regression was performed for the trend analysis of sentiment scores of positive and negative tweets during the COVID period. The significance level was set up as $p<0.05$.

\section{RESULTS}

A total of 36752 tweets gathered from Twitter social media platform. The 31352 unique Twitter users contributed to this total of 36752 tweets, whereas 3413 users sent multiple tweets. Sentiment analysis showed that tweets divided into negative $(n=8570)$, neutral $(n=15824)$, and positive $(n=12358)$. Figure $2 a$ presents an overview of new COVID-19 cases in Turkey between the start of 2020 and 14 May 2020 . There is a significant increase between the number of negative, positive, and total tweets in the COVID period when compared to the non-COVID period $(p=0.001)$ (Figure 2b).

The results, as shown in figure 3 , indicate that a statistically significant decrease Mean Sentiment Score during the COVID period when compared to the non-COVID period $(p=0.001)$. However, during the COVID period, no significant differences found between forced isolation days and non-forced isolation days for Mean Sentiment Score $(p=0.626)$ and the number of non-neutral tweets $(p=0.764)$. In figure 4, linear regression results showed a significant positive trend for Mean Sentiment Score of tweets within COVID period $(r 2=0.0064 ; p=0.04)$.

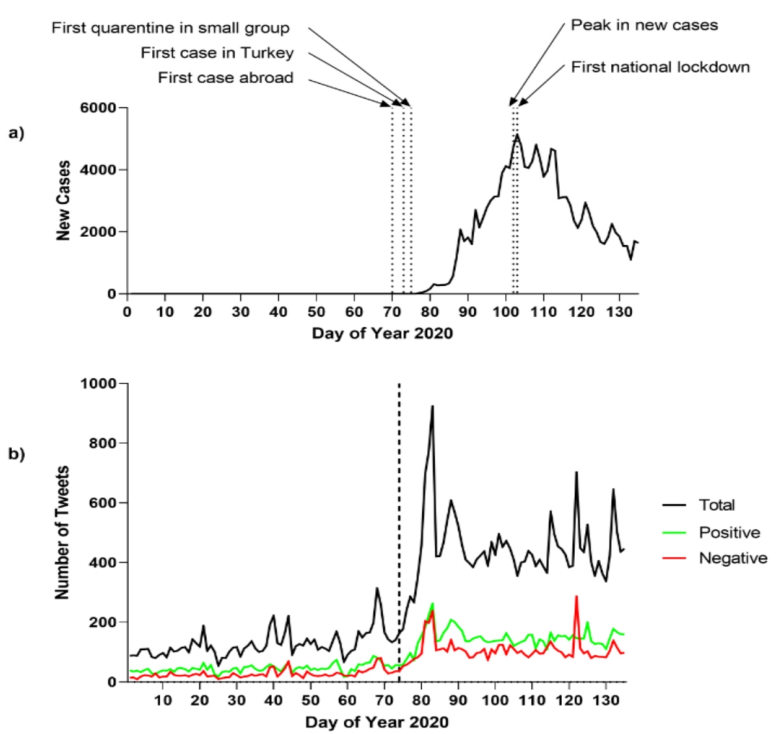

Figure 2. a) Distribution of new COVID-19 cases and some important days in the pandemic within the range of analyzed days in this study. b) The number of negative, positive, and total tweets within the range of analyzed days in this study. Positive tweets $n=3380$, negative tweets $n=2001$, and neutral tweets $n=3994$ in non-COVID period whereas positive tweets $n=8978$, negative tweets $n=6569$, and neutral tweets $n=11830$ in COVID period.

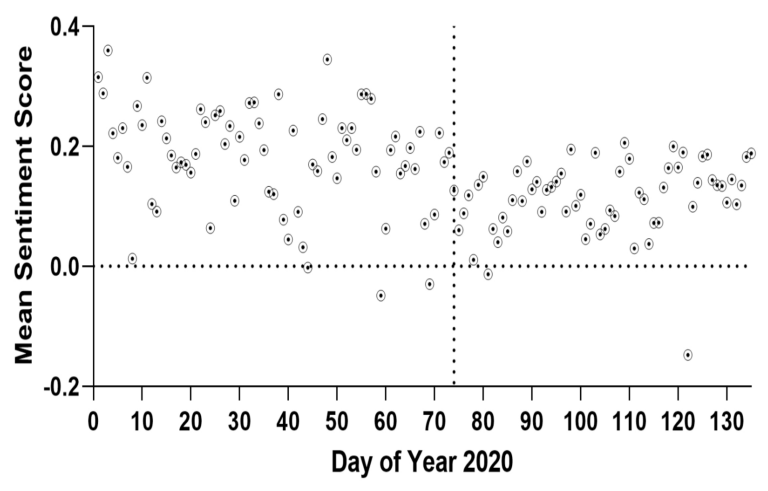

Figure 3. Mean Sentiment Score of tweets with separation of COVID and non-COVID period

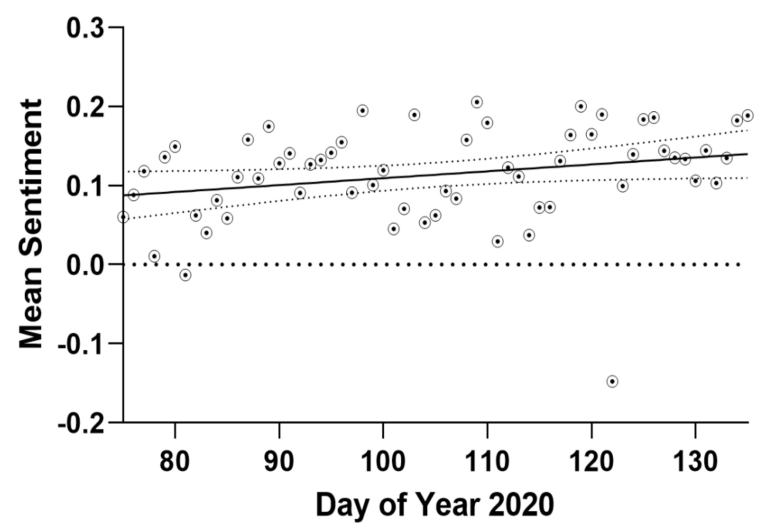

Figure 4 Linear regression analysis of Mean Sentiment Score of tweets within the COVID period. 


\section{DISCUSSION}

This study set out with the aim of analyzing the sentiments of social media in unprecedented times due to the COVID-19 pandemic. The finding of the present study indicates that Twitter usage increased due to the expression of being physically active during the initiate of the pandemic in Turkey. Our results showed that in the pandemic the sentiment analysis of tweets appears to play a key role to understand the attitude of tweets and effects of a physically inactive lockdown period.

The first finding of this study was a rising number of tweets and both positive and negative sentiments score of tweets in the COVID period in comparison to the non-COVID period. The observed increase in physical activity and exercise theme tweets could be attributed to escalating awareness of a healthy lifestyle. Not only having a chance to utilized more personal time but also incremental social media usage is likely to be related to a higher number of tweets. In addition, another possible explanation for this is that diminished energy expenditure and possible negative changes in nutritional behaviors (i.e. weight gain) resulted in an increase of individual exercising necessity within the COVID period. Likewise, improved number of being active tweets suggests that realization of the discontinuance the physically active opportunities such as fitness centers or gyms, schools' physical education classes and athletic training get meaning and directed to the exercise at home in the outbreak. Moreover, the government, institutions, universities, sports federations, popular teams and some celebrity athletes advised a be active message, organized an educational webinar in exercise topic, and shared basic exercise videos besides home stay recommendation. This promotional physical activity messages via Twitter seems related to an efficient way to direct into exercising for the public. These results corroborate the worldwide Twitter users according to April 2020 that observed Turkey places upper rankings (15). Furthermore, the health minister of Turkey declared the first positive infection of COVID-19 via Twitter and also efficiently used this platform during the pandemic (16). Consequently, there are some rapid reports and opinions emphasized that reducing the impact of physical activity during homestay prevention from new coronavirus. The statements of those conclusions were breaking often sedentary behaviors, decrease sitting time, maintain daily physical activity by household i.e. and exercise regularly $(4,11,17)$. Indeed, nowadays the sedentary behaviors and physical inactivity are considered as also pandemic which accepted responsibility for almost 3 million deaths per year (18). The increased expression of physical activity and exercise-related posts on Twitter during restricted quarantine seems to be consistent with the key study emphasized that hazardous effects on health (19). The study underlined the physical inactivity and sedentary behavior concerning the earlier isolation experiences in the literature, which concluded demand for investigations about long-term effects of mandatory isolation of COVID-19. Similarly, Werneck et al. found that an association with greater physical activity and lower sitting time and spent time TV watching in socially isolated children (20). Similar findings from the previous study confirm that COVID19 lockdown affected the lifestyles of the youth obese population because increased daily screen time on the other hand decreased the time participated in sports activities (21). Therefore, we needed to regarding children's and adolescents' risk of excess adipose tissue and weight gain, which is a barrier against healthy development in rare times a nonschool mass quarantine period.

Previous studies that noted the beneficial effects of social media to motivate physical activity including Twitter and Facebook platforms $(8,22)$. In these rare days in the shade of COVID-19 pandemic, several reports revealed that pandemic related social distance, isolations, and lockdown decreased the physical activity (6) while increased the cardiovascular disease risk factors $(10,23)$. Dos rei et al. examined whether exercise has positive effects on the mood by collecting and analyzing Twitter data. They found significantly fewer tweets expressed anxiety that users profile matched with regular exercise (24). However, the tweet data identification and classification method were different from our study. As our best knowledge, only one study was conducted to sentiment analysis of lockdown during COVID-19 (13). However, methods of sentiment analysis used in Barkur et al. study were sort out the \#IndiaLockdown and \#IndiafightsCorona not even close to the pair of current study' approach. Nevertheless, Cinelli et al. carried-out data analysis study about a social media infodemic related to COVID-19 (25). They examined the five big social media and found that Twitter was the most neutral 
one, whereas most sharing however second in users and comments. These results are in line with the current results that total tweets number did not provide the same users and physical trainer professions and sports companies.

The other findings of the current study were that a significant decrease in sentiment scores in COVID period when compared to non-COVID period, however, during the COVID period, no significant differences found between forced isolation days and non-forced isolation days for Mean Sentiment Score and the number of non-neutral tweets. It is possible to explain that the self-isolation policy accepted and efficiently performed in Turkey even the government forced isolation to the public. Nevertheless, the reason for this is not clear but it may have a relation with Twitter has not a real-time reflection on the declaration of isolation days. This delayed effect may partly explain by the positive trend of sentiments of tweets in the COVID period day after day. Interestingly, sentiments of tweets showed a positive trend in the COVID period from the beginning to the end of the lockdown. Contradict to current findings from Brooks et al., quarantine periods considered as a stressor factor which affect negatively the psychological as a result of disruptions of daily life (26). Similarly, Lima et al., drew attention to the negative emotional impacts of new coronavirus outbreak and lockdown (27). A possible explanation to both rise in negative and positive tweets maybe some of the people cannot have time and budget for exercise in their daily life and COVID lockdown favors the exercising at home whereas others have leisure time and access to the gym like facilities and cannot go anymore within the COVID period. The observed decrease in Mean Sentiment Score according to COVID period to non-COVID period could be explained by fear, anger, and anxiety that dominating negative mood based on the new coronavirus outbreak resulted in mortality, unemployment i.e. This also accords with a survey-based investigation by Zhang et al. (2020) that indicated people who get disruption to their work reports higher compliments of physical health whereas people who exercised more often at home affirm better life satisfaction (28). Hiremath et al. highlighted that importance of physical activity and regular exercise to overcome to adverse consequences of COVID-19 pandemic to mental health (29).
These results suggest that people had more leisure time and realized the importance of being active for a healthy lifestyle during this lockdown period in Turkey then both performed exercises and posted to Twitter more often. These increased number of tweets showed that exercising reflects an improved mood in their social media sharing.

\section{CONCLUSION}

This study limited with keywords that scanned to the tweets. Another limitation is our method of tweet classification underwent only double-edge one dimension (attitude) however, there are some other methods for multiple dimensions such as anxiety, anger, fear i.e. Future studies needed to observe to long-term impacts of the lockdown on our physical activity and exercise habits and reflections on social media. Further, the dataset of this study presented as public free open access (30). As a result of the dataset of the current study included temporal information, therefore it could be used in future studies within different queries and methods. In conclusion, this is an initial study that produced promising results for Twitter as a measurement tool for exercise and sports investigations. Current findings also showed that social media is a platform for promoting physical activity and exercise via peoples' necessity to share and get interaction.

Conflict of interest: There are no conflicts of interest in this paper. The authors whose names are listed in this paper certify that they have no affiliations with or involvement in any organization or entity with any financial interest or non-financial interest in the subject matter or materials discussed in this manuscript.

Funding: This study was not supported by any sources of funding. Peer-review: Externally peer-reviewed.

\section{REFERENCES}

1. Health Ministry RoT. COVID-19 information. https://COVID19bilgi.saglik.gov.tr/tr/2020 [cited 2020 11th June].

2. Burcu Tokuç GV. Medical Education in Turkey in Time of COVID-19. Balkan Medical Journal2020.

3. Gössling S, Scott D, Hall CM. Pandemics, tourism and global change: a rapid assessment of COVID19. Journal of Sustainable Tourism. 2020:1-20.

4. Haleem A, Javaid M, Vaishya R. Effects of COVID 19 pandemic in daily life. Curr Med Res Pract. 2020 Apr 3.

5. Cellini N, Canale N, Mioni G, Costa S. Changes in sleep pattern, sense of time and digital media use during COVID-19 lockdown in Italy. J Sleep Res. 2020 May 15:e13074. 
6. Hemphill NM, Kuan MTY, Harris KC. Reduced Physical Activity During COVID-19 Pandemic in Children With Congenital Heart Disease. Can J Cardiol. 2020 May 5.

7. Zhang J, Brackbill D, Yang S, Centola D. Efficacy and causal mechanism of an online social media intervention to increase physical activity: Results of a randomized controlled trial. Prev Med Rep. 2015;2:651-7.

https://doi.org/10.1016/j.pmedr.2015.08.005

8. Achen RM. Using Facebook and Twitter to Encourage Physical Activity: Are College Students Connecting with Campus Recreation on Social Media? A Pilot Study. Recreational Sports Journal. 2018;39(2):132-43.

9. Lippi G, Henry BM, Bovo C, Sanchis-Gomar F. Health risks and potential remedies during prolonged lockdowns for coronavirus disease 2019 (COVID-19). Diagnosis (Berl). 2020a May 26;7(2):85-90.

10. Lippi G, Henry BM, Sanchis-Gomar F. Physical inactivity and cardiovascular disease at the time of coronavirus disease 2019 (COVID-19). Eur J Prev Cardiol. 2020b Jun;27(9):906-8.

11. Chen $P$, Mao $L$, Nassis GP, Harmer $P$, Ainsworth BE, Li F. Coronavirus disease (COVID-19): The need to maintain regular physical activity while taking precautions. J Sport Health Sci. 2020 Mar;9(2):103-4.

https://doi.org/10.1016/j.jshs.2020.02.001

12. Batool R, Khattak AM, Maqbool J, Lee S, editors. Precise tweet classification and sentiment analysis. 2013 IEEE/ACIS 12th International Conference on Computer and Information Science (ICIS); 2013: IEEE.

13. Barkur G, Vibha GBKJAjop. Sentiment analysis of nationwide lockdown due to COVID 19 outbreak: Evidence from India. 2020. https://doi.org/10.1016/j.ajp.2020.102089

14. Harris JK, Duncan A, Men V, Shevick N, Krauss MJ, Cavazos-Rehg PA. Messengers and Messages for Tweets That Used \#thinspo and \#fitspo Hashtags in 2016. Prev Chronic Dis. 2018 Jan 4;15:E01.

15. Statista. Twitter: most users by country https://www.statista.com/statistics/242606/numb er-of-active-twitter-users-in-selectedcountries/2020 [16 June 2020].

16. Twitter. Tweet of Fahrettin Koca https://twitter.com/drfahrettinkoca/status/12383
36845131534336: Twitter, 13 March 2020 [cited 202016 June ].

17. Margaritis I, Houdart S, El Ouadrhiri Y, Bigard X, Vuillemin A, Duche P. How to deal with COVID19 epidemic-related lockdown physical inactivity and sedentary increase in youth? Adaptation of Anses' benchmarks. Arch Public Health. 2020;78:52.

18. Pratt M, Varela AR, Salvo D, Kohl III HW, Ding D. Attacking the pandemic of physical inactivity: what is holding us back?: BMJ Publishing Group Ltd and British Association of Sport and Exercise Medicine; 2019.

19. Hall G, Laddu DR, Phillips SA, Lavie CJ, Arena RJPiCD. A tale of two pandemics: How will COVID-19 and global trends in physical inactivity and sedentary behavior affect one another? 2020.

20. Werneck AO, Collings PJ, Barboza LL, Stubbs B, Silva DRJGhp. Associations of sedentary behaviors and physical activity with social isolation in 100,839 school students: The Brazilian Scholar Health Survey. 2019;59:7-13.

21. Pietrobelli $A$, Pecoraro $L$, Ferruzzi $A$, Heo $M$, Faith M, Zoller T, et al. Effects of COVID-19 Lockdown on Lifestyle Behaviors in Children with Obesity Living in Verona, Italy: A Longitudinal Study. Obesity (Silver Spring). 2020 Apr 30.

22. Cavallo DN, Tate DF, Ries AV, Brown JD, DeVellis RF, Ammerman AS. A social mediabased physical activity intervention: a randomized controlled trial. Am J Prev Med. 2012 Nov;43(5):527-32. https://doi.org/10.1016/j.amepre.2012.07.019

23. Mattioli AV, Ballerini Puviani M, Nasi M, Farinetti A. COVID-19 pandemic: the effects of quarantine on cardiovascular risk. Eur J Clin Nutr. 2020 May 5.

24. Dos Reis VL, Culotta A, editors. Using matched samples to estimate the effects of exercise on mental health via twitter. Twenty-Ninth AAAI Conference on Artificial Intelligence; 2015.

25. Cinelli M, Quattrociocchi W, Galeazzi A, Valensise CM, Brugnoli E, Schmidt AL, et al. The covid-19 social media infodemic. 2020.

26. Brooks SK, Webster RK, Smith LE, Woodland L, Wessely S, Greenberg N, et al. The psychological impact of quarantine and how to reduce it: rapid review of the evidence. 2020 . https://doi.org/10.2139/ssrn.3532534 
27. Lima CKT, de Medeiros Carvalho PM, Lima IdAS, de Oliveira Nunes JVA, Saraiva JS, de Souza RI, et al. The emotional impact of Coronavirus 2019nCoV (new Coronavirus disease). 2020:112915.

28. Zhang SX, Wang $Y$, Rauch A, Wei F. Unprecedented disruption of lives and work: Health, distress and life satisfaction of working adults in China one month into the COVID-19 outbreak. Psychiatry Res. 2020 Jun;288:112958.

29. Hiremath $P$, Suhas Kowshik CS, Manjunath $M$, Shettar M. COVID 19: Impact of lock-down on mental health and tips to overcome. Asian J Psychiatr. 2020 Apr 10;51:102088.

30. Physical Activity Tweets in Turkey during Covid19. [database on the Internet]. Kaggle. 2020. https://dx.doi.org/10.34740/kaggle/dsv/1245170 